\section{EMBnet - the European Molecular Biology Network, Moving forward: 2010 \& hevond}

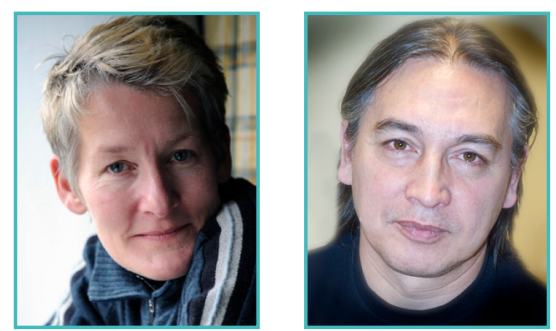

Teresa K. Attwood', Erik Bongcam-Rudloff ${ }^{2}$
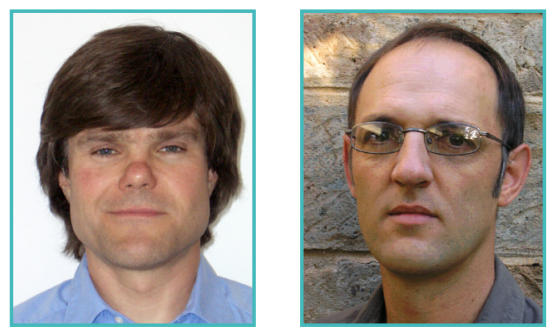

Andreas Gisel ${ }^{3}$, Etienne de Villiers ${ }^{4}$

1 Faculty of Life Sciences and School of Computer Sciences, University of Manchester, UK, ${ }^{2}$ The Linnaeus Centre for Bioinformatics, SLU/UU, SE, ${ }^{3}$ Institute for Biomedical Technologies, CNR, Bari, IT. ${ }^{4}$ EMBnet BecA-ILRI, Nairobi, KE

\section{Executive Summary}

The world that gave rise to EMBnet 22 years ago has changed; EMBnet has changed too. Initially focusing on shared European community needs, the organisation now embraces partners from around the world, whose aims, aspirations and communities are very different. Over time, its centre of gravity has shifted: no longer needed as a mechanism for distributing the EMBL databases, EMBnet has moved on. In the past, its activities have been funded by membership fees and a series of European grants; now, however, against a background of emerging (government-funded) European infrastructural initiatives, EMBnet's funding mechanisms need to be reviewed, in order to continue to sustain its core activities.

EMBnet is at a cross-roads. Before taking its next steps, it is appropriate to consider how the global bioinformatics landscape is evolving, and how EMBnet needs to adapt. This paper outlines a number of practical steps that could be taken, tempered by today's funding climate. Its principal recommendations are that EMBnet should:

i. review and properly define the roles, aims and goals of its Executive Board (EB) and Project Committees (PCs), and consider establishing additional PCs or Special Interest Groups (SIGs), with well-defined roles, aims and goals;

ii. review how EMBnet and its collection of PCs/ SIGs might achieve its goals, with or without further funding;

iii. identify and exploit its Unique Selling Point (USP);

iv. review and better understand who its communities are, what their needs are, and how to be more responsive to those needs;

v. review, streamline and clarify its current membership scheme;

vi. review how and why it might interact with other networks and organisations;

vii. establish internal infrastructures that would allow it to make strategic ties to other bioinformatics networks and organisations;

viii. establish internal infrastructures that would allow it to make more strategic responses to global funding opportunities;

ix. review the evolving role and internal structure of EMBnet.journal, and consider more tactical publishing strategies; and, in light of these considerations,

$x$. review and revamp its current name, brand and Website.

This paper is an open invitation for every member of the constituency to help with this critical evaluation of EMBnet's unique attributes and strengths; to consider how to build on these to create a competent, valuable and focused organisation that complements existing and emerging bioinformatics institutes, networks, associations and societies worldwide; ultimately, to maintain EMBnet's relevance in 2010 and beyond.

\section{Background}

Established in 1988, EMBnet served as an organisation for disseminating data, knowledge and services to support and advance research in molecular biology and biotechnology across a broad European community. Its service provision and knowledge sharing was primarily orchestrated by 'National Nodes' with government 
mandates to support their local communities; in time, the organisation also attracted a number of 'Specialist' and 'Industrial' Nodes, whose resources and know-how were seen to complement those of its National Nodes.

One of the major drivers for establishing EMBnet was the need for local access to data from centralised sources. In particular, it was intended to function as a distribution network for the EMBL Data Library databases (delivered, in those days, by floppy-disc and/or CD-ROM); consequently, from its inception, EMBnet had a special relationship with the EMBL. However, the growth of the EMBL nucleotide sequence database ultimately rendered this form of data distribution untenable; moreover, as local bioinformatics resources became more commonplace, with increasing compute power and swifter networks, reliance on the central facilities at EMBL was reduced or obviated. Eventually, the EMBL databases moved to the EBI outstation, when that was created in 1995.

Now, more than 20 years on, EMBnet has changed substantially. Not least, for more than a decade, the organisation has been embracing increasing numbers of countries from continents around the world - its constituency is hence no longer European, as illustrated in Fig. 1. Moreover, new research methodologies like 'Next Generation Sequencing' (NGS) are producing data on an unprecedented scale, and are once again driving the need for local services (processing, storage, management, analysis, software and database development, help and advice, etc.). Today, then, EMBnet's remit reaches far beyond the realms of support for molecular biology research.

EMBnet is in a state of transition. From the original handful of European Nodes, it has grown into an expansive international network of bioinformatics and biocomputing centres, the missions of which vary widely: from service centres specialising in scientific computing and communications, to those focusing on the molecular biology and biotechnology of plant, animal and insect viruses, and those dedicated to bioinformatics outreach and training. The nature and size of the local target communities vary enormously, and the range of service provision varies accordingly.

Over the years, one of EMBnet's most prominent developments has been its newsletter,

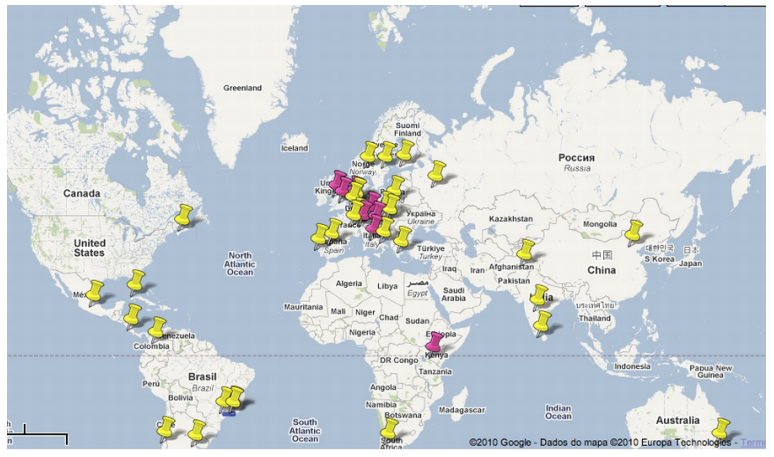

Figure 1: Snapshot of EMBnet Nodes in 2009, showing representation across the globe (yellow pins are National Nodes, pink are Specialist Nodes). The constituency is no longer European.

EMBnet.news. This has become a significant publication and, for many Nodes, provides an ideal way of reaching their local research communities with mission-relevant information: with its emphasis on presentation of everyday bioinformatics problems and their practical solutions, it fills a gap between bioinformatics theory and data management. Now, as part of EMBnet's expansion, the newsletter has begun to transition from a largely technical publication to a peerreviewed journal.

While EMBnet has been expanding across the globe, reaching out to wider and more diverse communities with each new Node joining the Network, major initiatives have begun to emerge to try to address the growing need for a European data infrastructure. Against this background, it is therefore timely to review where EMBnet came from and why, what its role is now in both European and global contexts, and how the organisation should move forward, for the benefit of its global membership.

\section{Perspectives on EMBnet}

In order to continue running its business beyond its first 5 years, EMBnet was established as a foundation (Stichting), registered in Nijmegen, The Netherlands, in 1993. To operate efficiently, EMBnet initially established 5 Project Committees (PCs), each with a minimum of 3 members: the PCs were i) Technical Developments; ii) Connectivity \& Software; iii) Information Services; iv) R\&D; and v) Workshops \& Training. These were overseen by a Steering Committee, also comprising a minimum of 3 members: a Chair (the spokesman and legal representative in official functions); a Treasurer (concerned with collec- 
tion and disbursement of funds); and a Secretary (concerned with keeping official records). Over time, there have been various changes to this structure, such that, today, EMBnet discharges its duties via 3 PCs (Education \& Training; Technical Manager; Publicity \& PR) and an Executive Board (EB).

In thinking about what EMBnet was and what EMBnet is today, it is helpful to consider how it has evolved, what its roles and goals should or could be moving forward, the steps needed to achieve them, and whether and/or how these might be funded. In the sections that follow, we reflect on these questions, both at an over-arching organisational level, and at the level of its internal structures - i.e., its PCs and EB.

\section{EMBnet, the organisation}

Current role, and its role and goals moving forward

The current role of EMBnet largely involves the following activities:

i. sharing data, knowledge and technological knowhow amongst its partners;

ii. acting as a portal for bioinformatics-related information;

iii. engaging its members in bioinformatics training activities;

iv. promoting the activities of its members via its online publication;

v. attracting new members to the Network.

Moving forward, EMBnet could build on these activities, to become more global and outward looking in terms both of sharing bioinformaticsrelated information and experience, and of stimulating and/or supporting training activities and capacity building.

Strategically, in the short term, EMBnet should aim to make a commitment both to seek collaboration/cooperation with other bioinformatics networks and organisations, and to seek funding for its future activities via opportunities worldwide.

In the longer term, it should aim i) to be a global hub of bioinformatics networks; ii) to host a range of funded research/training/capacitybuilding activities; and iii) to nurture its successful online publication as it evolves into EMBnet. journal.
Steps needed to achieve its goals

A) Steps towards short-term goals:

A number of immediate and fairly pragmatic steps could be taken to help EMBnet achieve its short-term goals. These include:

i. reviewing and properly defining the roles, aims and goals of its EB and PCs;

ii. reviewing its current structure, and considering whether additional formal PCs or perhaps more 'fluid' Special Interest Groups (SIGs) might be useful (e.g., for NGS discussions); and, if additional or different PCs or SIGs are considered beneficial, firmly defining their roles, aims and goals;

iii. reviewing what EMBnet as a whole, and as a collection of PCs/SIGs, can realistically achieve, with or without further funding;

iv. identifying and exploiting its USP!

V. reviewing and better understanding who its communities are (novice users, bench researchers, tool/resource developers, educators, etc.) and what their needs are, and being responsive to those needs;

vi. reviewing and streamlining its current membership scheme - e.g., clarifying the rules and goals of personal membership such that the scheme is a) widely understood, b) simple to implement, and hence, ultimately, c) widely used;

vii. reviewing how and why it might interact with other networks and organisations;

viii. establishing internal infrastructures that would allow it to make strategic ties to other bioinformatics networks and organisations (e.g., through a dedicated outreach PC and its nominated leader);

ix. establishing internal infrastructures that would allow it to make more strategic responses to global funding opportunities (e.g., through its SIGs and the designated leaders of those SIGs); and

xi. reviewing the evolving role and internal structure of EMBnet.journal, and considering more tactical publishing strategies (e.g., via themed issues, special conference or workshop proceedings; and so on).

\section{B) Steps towards longer-term goals:}

In order to meet its longer-term goals, once again, a number of relatively straightforward and 
pragmatic steps could be taken. These include, but are not limited to:

i. ensuring that the Website properly reflects EMBnet moving forward - e.g., with a meaningful name and 'brand', with an easy-to-use interface, with informative content, by embracing social networking technologies, and so on;

ii. applying for funding from a variety of different sources;

iii. joining global funding consortia.

As EMBnet moves forward, its internal and external communication mechanisms must work efficiently and effectively, and it should strive to work/cooperate closely with other organisations (ISCB, APBioNet, Bioinformatics.Org, the African Bioinformatics Network and so on.) and, above all, not in competition with them. It should also consider its relationship with international bioinformatics conferences, such as ISMB, ECCB, etc.

Importantly, we need to understand what's different about EMBnet - i.e., what is its USP (Or USPs)? Having identified its USP(s), we need to build on it (them)! For example, EMBnet has a growing number of Nodes from developing countries we need to understand their needs, and to focus efforts there; we need to ask what other networks or organisations currently do, or plan to do, for these countries (and what they will not do); and we also need to understand the role of EMBnet. journal in this context.

Funding requirements and funding mechanisms

There is little here that needs direct funding (aside from a new Website), as the ideas primarily concern the need for internal structural changes within EMBnet. Nevertheless, realising EMBnet's ambitions will require identifying concrete courses of action, identifying strategic projects and key individuals (champions) who are prepared to help deliver them; moreover, it will require identifying the funding opportunities that exist to make these courses of action/projects possible, and will require concerted efforts to apply for those funds.

In terms of potential sources of funding, these are wide and varied. They might include, but are not limited to: the EU; other national and international funding bodies (e.g., for capacity building, and so on); Bill Gates Foundation; etc.

\section{The Executive Board}

The following sections outline the EB's view of its role, aims and goals, set in the context of a vision and mission for EMBnet as a whole, moving forward from 2010.

Everything outlined here hinges on the people within EMBnet, their willingness to support a collective vision and their enthusiasm to act upon it. Our specific, and most urgent, recommendation is the development of a new Website.

Current role, and its role and goals moving forward

The role of the EB is to carry out the decisions of the Board and to run the daily business of EMBnet. It currently works towards achieving this, in close collaboration both with its PCs and with the full Board, in two main ways: i) formally, through monthly Virtual General Meetings (VGMs) and Annual General Meetings (AGMs), and ii) informally, via its email lists. In particular, the EB is responsible for managing the Stichting financial accounts, and for preparing an annual financial report for discussion at its AGMs.

As EMBnet moves forward, the EB should aim to provide vision, leadership and efficient executive coordination of the activities of the PCs and of the growing number of Nodes (and possibly of SIGs, should it establish them).

In the short term, the EB aims to inculcate a spirit of professionalism, dignity and collegiality amongst members of the Board in general, and of the PCs in particular, so that EMBnet can conduct its future business effectively and efficiently.

In the longer-term, the EB should aim to establish internal mechanisms by means of which i) it might secure strategic alliances with other organisations, ii) its members might compete more strategically for funding to support some of its activities, and iii) it might promote its activities more effectively.

\section{Steps needed to achieve its goals}

A) Steps towards short-term goals:

A number of immediate and practical steps could be taken to help the EB achieve its shortterm goals. These include:

i. properly defining the roles of EB members (of the Chair, Secretary, Treasurer, etc.), and helping to create closer ties between Board members and PCs/SIGs;

ii. helping to promote the establishment of strategic alliances with other networks; 
iii. rationalising how VGMs and AGMs are conducted, ensuring that the groundwork for these meetings is prepared in advance;

iv. formalising nomination procedures, so that voting at AGMs (and/or VGMs) is properly informed;

v. helping to improve the EMBnet brand.

\section{B) Steps towards longer-term goals:}

In order to meet its longer-term goals, again, various simple practical steps could be taken. These include:

i. discussing within the EB, with the PCs and the wider EMBnet constituency, the establishment of SIGs, whose role could include, amongst other things, the coordination of members with mutual interest in particular funding calls;

ii. encouraging and supporting the improvement/re-design of the Website;

iii. encouraging better use of the Website or, rather, helping to make the Website work better for EMBnet.

Funding requirements and funding mechanisms

Most of these ideas do not have direct funding implications per se, as they primarily concern the need for internal structural changes. However, redevelopment of the Website may have an associated cost, which could probably be met by the Stichting.

\section{The Education \& Training PC}

Current role, and its role and goals moving forward

The E\&T PC is in a state of flux, but is trying to focus its efforts on producing new, and updating old, Quick Guides (QGs). Importantly, it can cover the whole QG-publication workflow, relieving the P\&PR PC of this process.

The E\&T PC should strive to be a strong, wellstructured course-giving PC. It should be able to organise tutorials and workshops, to provide training to local communities through its network of Nodes. The courses could range from basic to advanced topics, and could be delivered in a systematic way: e.g., it could start with 2 courses per semester in different locations, eventually becoming a fund-raising activity for EMBnet. With financial support from local and international donors, the E\&T PC could provide both the nec- essary infrastructure and the course materials. As part of its goal to provide such training to communities throughout the world, the E\&T PC could champion the production of 'EMBnet Kits'. For example:

i. EMBnet Kits for students. In order to disseminate bioinformatics history, data, methods, resources, and so on, a student kit, including a professionally produced set of carefully selected materials (texts, videos, training datasets, free programs, etc.), could be distributed via DVD, USB stick, etc. The kit could be offered (or sold) to Universities and Institutes for distribution to students - this could be done, for example, in collaboration with initiatives like SLING;

ii. EMBnet Kits for medical professionals. A similar resource could be produced for medical practitioners, many of whom are unaware of the benefits of accessing biological data and core bioinformatics methodologies. A kit with illustrated examples could help to motivate medical professionals to look at problems with different eyes, and could have the added benefit of bringing new projects to bioinformaticians, and hence of initiating new collaborations. 'Bio-data aware' medical researchers and practitioners are sorely needed.

\section{Steps needed to achieve its goals}

In order to achieve its goals, a number of steps are necessary. The E\&T PC should:

i. better define its role and the roles of its members;

ii. define the scope and parameters of its courses, especially if it is to embark on travelling workshops/road-shows to different international locations;

iii. cooperate closely with people outside the $\mathrm{PC}$, to ensure the delivery of relevant, triedand-tested courses and tutorials;

iv. keep all of its materials up to date, if it is to deliver useful courses; and

v. cooperate with the P\&PR PC to produce and promote EMBnet Kits.

Funding requirements and funding mechanisms

Organising courses requires funds (e.g., to cover tutors' expenses). National and international funding donors need to be identified (e.g., bio- 
informatics companies, Marie Curie grants). The possibility of co-organising courses with other organisations (e.g., FEBS, EMBO) could also be considered.

\section{The TM PC}

Current role, and its role and goals moving forward

The role of the TM PC is to:

i. provide the technical foundation for EMBnet's information flow: this encompasses DNSservices, mail-list services, a Marratech video conferencing system and, most importantly, maintenance of a Web server and its subsystems (Drupal, OJS, etc.);

ii. provide guidance to Node managers and scientists around the world about setting up IT infrastructure resources to facilitate bio-computing applications (including help with technical specifications, service/software set-up issues, and so on);

iii. maintain expertise in the development and maintenance of key applications in the field of biological sequence analysis and retrieval systems, including open source applications such as MRS; wEMBOSS; MIRA 3 Whole Genome Shotgun and EST Sequence Assembler for Sanger, 454 and Solexa/ Illumina; Galaxy workflows; Taverna; tools that facilitate formatting/indexing of flat-file databases;

iv. provide technical advice and investigations for core life science computing problems (e.g., for NGS data storage and processing resources).

In addition to maintaining and developing EMBnet's existing information management infrastructure, the TM PC would like to support the following emerging projects:

i. technical challenges involving the storage and pipeline construction of NGS data;

ii. GPU computing paradigms in the life sciences.

In the short-term, we would like to deploy improved backup and mirror systems for the www. embnet.org site. We recommend that both the existing and mirror sites should move to a Linux Open Source platform, as MacOS X presents problems when compiling various open source packages. In the longer term, the TM PC would seek to become a point of reference for technical bio-computing issues.

\section{Steps needed to achieve its goals}

To achieve our short-term goals, we seek a modest annual budget to be allocated to various projects and consumables (data drives, memory, software) needed to sustain the basic services. A Mac Mini server is set in Pakistan. Help/guidance in mirroring the EMBnet site will be needed.

Steps required to achieve our long-term goals are more challenging:

i. the capacity of the present server is unlikely to be sufficient for much longer, and should therefore be upgraded within the next year;

ii. the issue of Marratech vs other video conferencing solutions might be solved by using freely available university resources;

iii. the TM PC could become involved with exploratory projects, looking at emerging IT- or scientific developments/trends in partnership with particular Nodes. This would be mutually beneficial to the Node(s) and to EMBnet;

iv. sharing our expertise via a blog could be useful, and could help to stimulate wider participation in EMBent. A FAQ-list could be assembled, which, if structured and equipped with a navigation tool, could be useful.

Funding requirements and funding mechanisms

Upgrading the systems, subscribing to a backup service, and projects involving long-term support will need external funding. Projects of general interest could be presented to emerging 'bigscience' bio-projects, such as the Science for Life Laboratories in Stockholm and Uppsala, and corresponding initiatives in other countries.

The P\&PR PC

Current role, and its role and goals moving forward

The main roles of the P\&PR PC are to:

i. nurture and promote EMBnet's image at large, enhancing its visibility;

ii. promote EMBnet and its activities by:

- maintaining and enhancing the Website;

- supporting the publication and dissemination of EMBnet.journal;

- liaising with other groups and societies;

- fostering appropriate, active connections with ISCB, APBioNet, etc. 
Future roles could include:

i. establishing effective links with trans-national organisations that have vested interests in the area, such as the Ludwig Institute for Cancer Research, Sloan Kettering, GBIF (Global Biodiversity Information Facility), TDWG, for example;

ii. helping to devise new mechanisms for attracting sponsors for EMBnet.journal, and for courses, workshops, conferences, etc.

The goals of the P\&PR PC include bootstrapping a number of short- and long-term projects and initiatives, including, for example:

i. Re-designing the Website. The new site needs to be structured to include more informative content and to be more easy-to-use: e.g., it should showcase: EMBnet's people, partners and institutes; its services and resources; its National, Associate and Specialist Nodes; its widely distributed activities; etc.;

ii. Geo-referencing EMBnet. This could be seen either as an addition to the Website (and made available for inclusion in other Websites) or as an alternative view of EMBnet. It should provide an up-to-date overview of the organisation and allow people to readily contact their nearest Node;

iii. Social networking. EMBnet's Linkedln group could be used to hold discussions, to generate news feeds and to open new discussions.

All of these initiatives will require help and collaboration with:

- the EB, in decisions relating to public relations and cooperation with other related groups, networks and societies worldwide;

- the E\&T PC, to support them in the promotion, organisation and dissemination of QGs, training courses and materials, summer schools, and so on;

- the TM PC, in all issues related to the improvement of EMBnet's Website.

Funding requirements and funding mechanisms

There will be costs associated with printing and disseminating publicity materials. Promotion of EMBnet at conferences will be expensive, but if included in a cooperation agreement, say with other related networks and societies, the costs could be reduced.

\section{Conclusion}

The world that gave rise to EMBnet 22 years ago has changed. As EMBnet has acquired more partners, its operational model has moved from a focus on shared European community needs, to one that embraces disparate Nodes with different aims and aspirations, and serving very different communities with very different requirements. As its partners moved on from their reliance on EMBnet as a mechanism for distributing the EMBL databases and E-GCG/EMBOSS, the organisation moved on too.

Today, it is important for EMBnet to try to understand how the global bioinformatics landscape is evolving, and how it should adapt. This paper has outlined some practical steps that could be taken, all of which need to be tempered by a proper understanding of the current funding climate. Ultimately, the paper is a call to arms: to embrace every member of the constituency in a critical evaluation of EMBnet's unique attributes and strengths; to consider how to build on these to create a focused organisation that complements existing and emerging bioinformatics institutes, networks, associations and societies worldwide; ultimately, to maintain EMBnet's relevance in 2010 and beyond. 